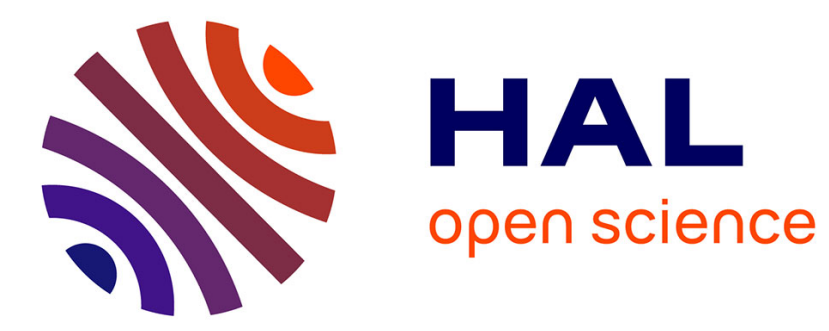

\title{
Condition Assessment and Fault Prognostics of Microelectromechanical Systems.
}

Kamal Medjaher, Haithem Skima, Noureddine Zerhouni

\section{To cite this version:}

Kamal Medjaher, Haithem Skima, Noureddine Zerhouni. Condition Assessment and Fault Prognostics of Microelectromechanical Systems.. Microelectronics Reliability, 2014, 54, pp.143-151. 10.1016/j.microrel.2013.09.013 . hal-00929738

\section{HAL Id: hal-00929738 \\ https://hal.science/hal-00929738}

Submitted on 13 Jan 2014

HAL is a multi-disciplinary open access archive for the deposit and dissemination of scientific research documents, whether they are published or not. The documents may come from teaching and research institutions in France or abroad, or from public or private research centers.
L'archive ouverte pluridisciplinaire HAL, est destinée au dépôt et à la diffusion de documents scientifiques de niveau recherche, publiés ou non, émanant des établissements d'enseignement et de recherche français ou étrangers, des laboratoires publics ou privés. 


\title{
Condition Assessment and Fault Prognostics of Microelectromechanical Systems
}

\author{
K. Medjaher*, H. Skima, N. Zerhouni \\ FEMTO-ST Institute, UMR CNRS 6174 - UFC / ENSMM / UTBM \\ Automatic Control and Micro-Mechatronic Systems Department \\ 24, rue Alain Savary, 25000 Besançon, France
}

\begin{abstract}
Microelectromechanical systems (MEMS) are used in different applications such as automotive, biomedical, aerospace and communication technologies. They create new functionalities and contribute to miniaturize the systems and reduce their costs. However, the reliability of MEMS is one of their major concerns. They suffer from different failure mechanisms which impact their performance, reduce their lifetime and their availability. It is then necessary to monitor their behavior and assess their health state to take appropriate decision such as control reconfiguration and maintenance. These tasks can be done by using Prognostic and Health Management (PHM) approaches. This paper addresses a condition assessment and fault prognostic method for MEMS. The paper starts with a short review about MEMS and presents some challenges identified and which need to be raised to implement PHM methods. The purpose is to highlight the intrinsic constraints of MEMS from PHM point of view. The proposed method is based on a global model combining both nominal behavior model and degradation model to assess the health state of MEMS and predict their remaining useful life. The method is applied on a microgripper, with different degradation models, to show its effectiveness.
\end{abstract}

Keywords: MEMS, Condition monitoring, Condition assessment, Fault detection, Fault diagnostics, Fault prognostics.

\footnotetext{
*Corresponding author. Tel.: +33 (0)3 81402796

Email address: kamal.medjaher@ens2m.fr (K. Medjaher)
} 


\section{Introduction}

Prognostics and Health Management (PHM) is a process involving different tasks. The purpose is to monitor systems, assess their health state, detect and diagnose their faults, anticipate the time to failures by calculating the remaining useful life (RUL) and take appropriate decisions accordingly. PHM is widely applied on industrial systems ranging from small components (bearings, gearboxes, batteries, etc.) to complete machines (wind turbines, electrical motors, machining tools, etc.). Several research works have been published on PHM in the recent decade, where different methods, algorithms and formalization tools are proposed $[1,2,3,4]$. The reported works can be grouped in three categories: model-based prognostics (also called physics of failure prognostics), data-driven prognostics and hybrid prognostics. The first approach uses first principles to derive behavior models and use them to estimate the current state of systems and predict their RUL. The second approach is based on the transformation of monitoring data into relevant models including the degradation of the system and use them to do PHM. Finally, the third approach combines both previous approaches.

PHM can be applied to microsystems, particularly to microelectromechanical systems (MEMS), since these latter devices undergo failures during their lifetime. However, the miniaturization of these systems introduces some specificities and constraints to take into account during PHM implementation. Among these constraints, two of them are particularly interesting: the first concerns MEMS monitoring and the second their degradation models. One way of getting relevant data about failure mechanisms which may take place in MEMS is to incorporate appropriate sensors within their structure. This means that the PHM functions should be considered from the design phase of MEMS. Concerning the degradation models, they can be obtained by two different ways: by using the experts' knowledge or by doing accelerated life tests. However, the second option can not be applied on all types of MEMS due to the fact that the activation of the acceleration factors (temperature,

humidity, stress, etc.) excites additional failure mechanisms and rends the degradation of MEMS difficult to model.

MEMS are designed to achieve specific functions in particular conditions. However, in practice and during their utilization, it is not uncommon to observe a gap between their reliability estimated during the design phase and their real reliability. This difference can be due to two factors: the fabrication process and the real operating conditions. To improve the reliability 
and availability of systems where MEMS are utilized, and also to reduce the number of accidents and maintenance costs, one of the possible solutions can be the implementation of PHM tasks.

This paper presents a condition assessment and fault prognostic method for MEMS. The paper first addresses a review of main works reported in the domain of condition monitoring, health assessment and reliability prediction of MEMS. This part deals also with main failure mechanisms in MEMS and the methods used to identify the roots of these phenomena. The paper presents then the proposed method and its steps. The method aims at assessing the health state of MEMS and estimate their RUL. It is based on a global model combining both nominal behavior model and degradation model of MEMS. The nominal model is obtained by using fundamental laws of physics whereas the degradation models can be generated by two ways: by running different simulations regarding linear and nonlinear models or through accelerated life tests performed experimentally. In this paper, and in order to validate the concept of PHM on MEMS, the simulation case is considered. However, to be close to real conditions, the parameters of the nominal behavior of the studied MEMS are identified from experimental measures.

The paper is organized in five sections. After the introduction, the second section presents a literature review related to MEMS reliability. The third section deals with the proposed method and its steps. The fourth section concerns the application of the method on a real electrostatic MEMS consisting of a microgripper and finally, section five concludes the paper.

\section{MEMS reliability review}

\subsection{State of the art}

MEMS are more and more utilized in different applications as summarized in figure 1. They can be grouped in two main categories [5]: MEMS with moving parts and MEMS without moving parts. However, almost all MEMS contain micro-structures and micro-elements or parts made from silicon. These parts are subject to degradations and failure mechanisms due to several factors (temperature, humidity, vibration, moisture, noise, electrical overloads, etc.). Common failure mechanisms identified and known until now concern stiction, wear, fracture, crystallographic defect, creep and degradation of dielectrics $[6,7,8,9]$. The reliability of these microsystems is then considered as a major obstacle in their development [10]. In practice, failures undergone by MEMS can decrease their reliability, availability 
and increase their exploitation costs. Moreover, in some applications these failures can lead to accidents and decrease the MEMS's performance as they prevent achieving the functions for which they are initially designed.

Several research works dealing with MEMS reliability have been reported

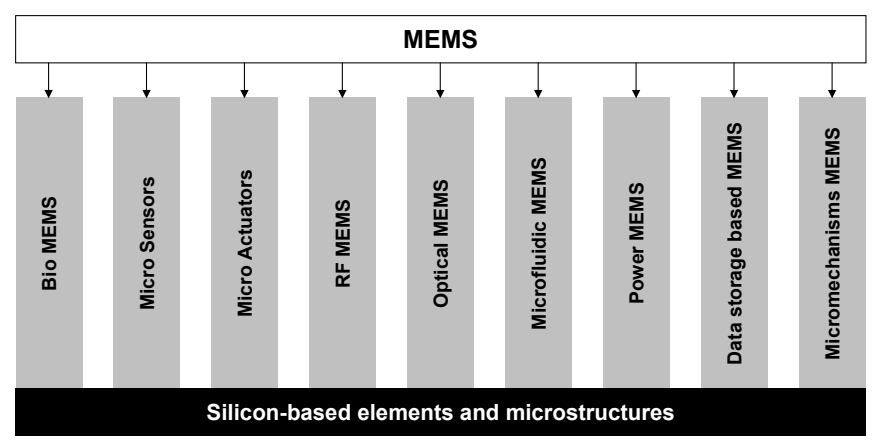

Figure 1: MEMS applications.

in the literature. These works can be classified into different categories: reliability reviews, accelerated life tests to understand the failure mechanisms, fault detection and diagnostics and fault prognostics (or time to failure estimation). These categories and related works are summarized in table 1. More details about each category are given in the following.

\subsubsection{Reliability reviews}

Huang et al. [6] reviewed common failure mechanisms in MEMS, inspection techniques and approaches to mitigate those failures through structure optimization and material selection. Similarly, Zaghloul et al. [7] reviewed the knowledge related to critical failure mechanisms (dielectric charging and stiction) in electrostatic micro and nano-electromechanical systems (MEMS and NEMS). The authors described the employed nanoscale characterization techniques for these failure mechanisms and presented new characterization methods used to correlate between the results from MEMS devices and metalinsulator-metal (MIM) capacitors. The paper presented in [8] deals with an overview of MEMS failure mechanisms that are commonly encountered (stiction, creep, fatigue, brittle fatigue in silicon, wear, dielectric charging, breakdown, contamination and packaging). The paper addresses also the reliability issues of micro-scale devices. Always in the same category, a review of MEMS reliability issues and failure mechanisms can be found in [13]. The chapter published in [9] reported known and unknown failure modes of 


\begin{tabular}{|c|c|c|}
\hline Review category & Addressed topics & References \\
\hline Reliability review & $\begin{array}{l}\text { - Common failure mecha- } \\
\text { nisms } \\
\text { - MEMS reliability issues }\end{array}$ & $\begin{array}{l}{[6,5,7,11,} \\
8,12,13,9]\end{array}$ \\
\hline $\begin{array}{l}\text { Condition assessment, } \\
\text { fault detection and di- } \\
\text { agnostics }\end{array}$ & $\begin{array}{l}\text { - Accelerated life tests } \\
\text { - Condition monitoring } \\
\text { - Characterization of fatigue } \\
\text { properties } \\
\text { - Fault detection (fatigue, } \\
\text { crack, damage, etc.) } \\
\text { - Root cause analysis }\end{array}$ & $\begin{array}{l}{[14,15,16,} \\
17,18,19, \\
20,21]\end{array}$ \\
\hline Fault prognostics & $\begin{array}{l}\text { - Reliability prediction } \\
\text { - Time To Failure (TTF) es- } \\
\text { timation }\end{array}$ & $\begin{array}{l}{[22,23,24} \\
10,25,26]\end{array}$ \\
\hline
\end{tabular}

Table 1: MEMS reliability review.

MEMS devices. The reliability of MEMS devices packaging is addressed in [27]. Tanner [5] reviewed the significant successes in MEMS products from reliability perspective and discussed the reliability concerns of various devices. The paper published in [11] presents the progress in reliability research in the micro and nano domains, where thermo-mechanical reliability of microelectronic components and systems and the related methods to analyze and predict it are addressed. Finally, in [12] the authors studied the mechanical response of a large class of shock loaded microsystems. The purpose of this study is to formulate some guidelines for design of reliable MEMS. 


\subsubsection{Condition assessment, fault detection and diagnostics}

Chen et al. [14] developed a fault detection method based on electrical analysis for valveless peristaltic lead zirconate titanate (PZT) micropump fabrication. In their method, the detection of PZT cracking, uneven silver epoxy distribution and PZT inversion failures is based on the utilization of a modified Butterworth-Van Dyke (BVD) model to analyze the properties of resistant or capacitive elements related to the three previous failures. The series resistance and parallel capacitance in the BVD model are used to detect faults and classify the failure types. In [15], the authors proposed a method for on-chip fatigue and fracture tests and monitoring on polysilicon specimens. The method is then tested on a setup designed to monitor the elastic stiffness of MEMS. The results of this work allowed to put in evidence the decrease of the elastic stiffness during fatigue life before rupture. The paper published in [16] summarizes testing methods for the characterization of fatigue properties of thin metal films used in MEMS. The tests and analysis conducted on different film thicknesses revealed that the fatigue and cracking behaviors are different from that of bulk materials, and are controlled by the length scales of the thin metal films (surface to volume ratio). In [17], a fault detection method for MEMS is presented. The method combines two modeling tools: a Competitive Neural Network (CNN) and a Robust Heteroscedastic Probabilistic Neural Network (RHPNN). The simulation results conducted by the authors showed that the fault detection percentage increases when combining both tools compared to the utilization of the RHPNN alone. In his paper [18], Walraven addressed the issues identified when performing root cause failure analysis of packaged, capped, and sealed MEMS devices. Mellé et al. [19] investigated the dielectric charging failure mechanism in capacitive RF-MEMS, which is caused by the leakage current through the RF-MEMS dielectric. The dynamic of the identified failure mechanism is then monitored through two parameters: the shift rate of the actuation voltages and a reliability-driven electrical stress parameter, which is based on the contact quality between the bridge and the dielectric. The paper published in [20] addresses the damage in polysilicon microstructures under fatigue loading cycles. In this work, the damage in the material is investigated by means of an elastic stiffness decrease monitoring during fatigue life, obtained through a low noise, low perturbing capacitive position readout of the MEMS moving mass. Finally, a method for fault testing in MEMS is proposed in [21] to detect the presence of faults in MEMS. 


\subsubsection{Reliability assessment and prediction}

This category of works can be also assimilated to the fault prognostic task as it deals with the estimation of the time to failure. Among these works, McMahon and Jones [22] proposed an accelerated life tests for MEMS to assess their reliability. The method investigated the use of mechanical means to actuate MEMS membranes so that lifetime estimates could be obtained. In their work, the authors have adapted a Talysurf measurement system to allow a MEMS membrane to be cycled by moving it with a stylus. This cycling was continued until the membrane failed according to the definitions provided by the device manufacturer. This experiment was repeated with different forces and this allowed standard life testing techniques to be used to produce a prediction of lifetime under normal use conditions. Matmat et al. [23] studied the effect of dielectric charging on the reliability of capacitive RF MEMS switches. The authors proposed a model to estimate the time to failure (TTF) of the RF MEMS switches. The proposed model takes into account the influence of the actuation bias signal, the duty cycle and the temperature on the degradation (consequently on the reliability) of the studied components. Ruan [24] reviewed the issues of reliability and accelerated life testing for radio frequency micro-electromechanical system (RF-MEMS) capacitive devices. Matmat et al. [10] suggested a method to estimate the lifetime of RF MEMS capacitive switch devices by combining functional and physical failure analysis. The physics of charging effects along with mechanical behavior of the membrane are introduced simultaneously to determine the time to failure of the RF MEMS devices. Tanner et al. [25] presented a new method to estimate the MEMS reliability. This method is based on modeling, simulation and material science, which is different from traditional reliability methods usually performed on mass production. The reliability method relies on four tasks, which are: (a) design, model and fabricate, (b) test structures and devices, (c) identify failure modes and mechanisms, (d) develop predictive reliability models (accelerated aging), and (e) develop qualification methods. Finally, the paper of Driel et al. [26] deals with reliability prediction of MEMS packages made from pieces of silicon placed on the top of the devices to protect them from external loads. The method presented by the authors combines parametric finite element models with experimental verifications to address the reliability of four different capping concepts and understand some capping issues (cavity deflection, cap fractures and moisture penetration). 


\subsection{Beyond the state of the art}

From the previous state of the art, one can distinguish two approaches: the first concerns MEMS reliability and the second is related to some tasks of PHM shown in figure 2 (monitoring, health assessment, fault detection and diagnostics and fault prognostics). The reliability aspects depend strongly on

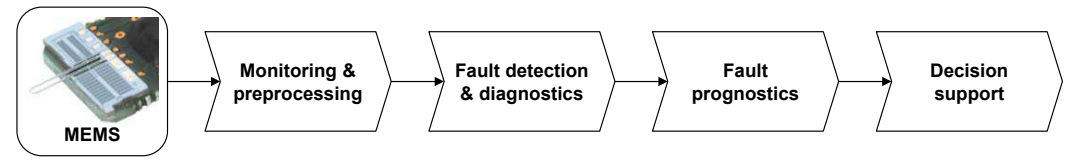

Figure 2: PHM main tasks.

the design of MEMS and on how their utilization is close to the specifications defined during the design phase, which rarely hold in practice. Therefore, some tasks of PHM can be implemented on MEMS to track their health state during their operation and act in case of abnormal behavior or functioning. The purpose is then to continuously monitor the behavior of MEMS, assess their current condition, predict their future state and estimate their remaining useful life (RUL). The information gathered by these tasks can then be exploited to take appropriate decisions to achieve the expected functions and improve the MEMS reliability and availability. The decisions can concern reconfiguration of control laws, set point changes, fault tolerant strategies, etc. Nevertheless, some challenges should be raised before implementing the above mentioned PHM tasks on MEMS. They are summarized in the following.

1. Sensing: contrary to macro-systems for which the installation of additional sensors to monitor their behavior and their degradations is possible, the case of MEMS is more constraining. The challenge concerns then two aspects: 1) defining and integrating appropriate sensors from the design phase for new MEMS to allow PHM, and 2) defining the needed sensors and the way of doing measurements in case of MEMS yet in service.

2. Behavior model: MEMS involve several micro parts and elements working with different types of energy (mechanical, electrical, thermal, hydraulics, etc.) and operating under different conditions (temperature, humidity, pressure, etc.) which make their behavior complex and strongly nonlinear. Thus, a trade-off must be found between complexity and simplicity of the model to be used for PHM. 
3. Accelerated life tests: the implementation of PHM methods is dependent on degradation models of the parts involved in MEMS subject to failures. These models can be obtained theoretically by using physical first principles and simulations or experimentally through accelerated life tests. The first approach requires deep understanding of failure mechanisms and may lead to models which can be difficult to use. The second approach can give models which take into account the operating conditions, but its practical implementation may be difficult due to several factors (repeatability of the tests, difficulty to install the required sensors, influence of the environmental parameters such as dust, temperature, humidity, etc.).

4. Degradation models: this challenge is partly dependent on the previous one and concerns the validation of the obtained degradation models and their utilization for PHM. Indeed, the degradation models obtained either theoretically or experimentally must remain valid within the framework of the studied MEMS. These models are then used with the nominal behavior models obtained previously to assess the MEMS conditions and estimate their RUL.

5. Failure thresholds: in addition to nominal behavior models and degradation models, fault thresholds are important. They define the acceptable performance limits of MEMS and allow their RUL calculation.

The following section deals with a condition assessment and RUL estimation method applied to a microgripper. The purpose is to show the feasibility of some tasks of PHM on MEMS by raising some of the above challenges, particularly those from the second to the fifth.

\section{Condition assessment and RUL estimation of MEMS}

The steps of the proposed method are summarized in figure 3 and explained below. The method can be applied on different categories of MEMS if the following assumptions are considered.

1. Appropriate sensors are available to monitor the behavior of MEMS.

2. Sufficient knowledge is available about the studied MEMS to derive its behavior models and identify the failure mechanisms which may take place during its utilization. 
3. Possibility to obtain the degradation models of the studied MEMS, either experimentally through accelerated life tests or theoretically by experts.

4. Sufficient knowledge is available to define the failure thresholds.

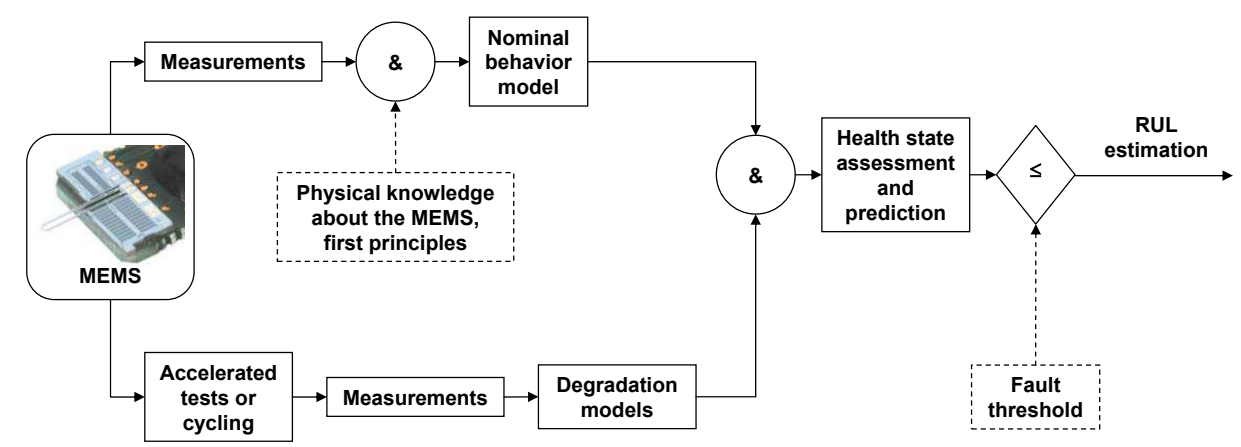

Figure 3: Main steps of the proposed method.

- MEMS: the targeted device can concern all the categories shown in figure 1. However, for each MEMS, it is important to have a deep understanding about its physical and failure phenomena and also about the environment in which it evolves. This knowledge will be useful for the next steps.

- Measurements: they are required to define the numerical values of the MEMS parameters, analyze its behavior (time and frequency responses, stability, precision, etc.), define the parameters involved in its failure mechanisms identified previously, define the degradation models and the fault thresholds. The measurements are provided by sensors which depend on the physical quantities to monitor, which in turn depend on the targeted MEMS. Examples of sensors are: interferometers to measure the displacement, force sensors, temperature sensors, accelerometers, etc. Moreover, relevant features which can be used to assess the health state of each MEMS and calculate its RUL can be extracted from these measurements. Examples of features are: time response, rising time, overshoot, stability, precision, etc.

- Nominal behavior model: it can be obtained by writing the physical equations (first principles) of the targeted MEMS or derived experimentally. The model can be linear or nonlinear. Its complexity depends also on the 
modeling assumptions made during its construction. The parameters of the model can be obtained from the manufacturer's specifications or identified by exciting the MEMS and getting its time or frequency response.

- Degradation models: they can be given by experts of the MEMS or obtained experimentally through accelerated life tests. In the case of models obtained experimentally, several MEMS must be tested under different operating conditions which should be as close as possible to those of the real utilization of the MEMS. The degradation models obtained from the tested MEMS must be also representative of those used in practice. These models can be related to drifts of the physical parameters of MEMS (mass, friction coefficients, stiffness, etc.), and can be obtained by analyzing the data acquired from the accelerated life tests and by using appropriate modeling tools (regressions, curve fittings, etc.).

- Health assessment and prediction: this task is performed by combining both nominal and degradation models of the targeted MEMS. The global model is then used to estimate the state of the MEMS, compare this state to the performance threshold (or fault threshold) and calculate the RUL. This task may also concern fault detection and diagnostics of abrupt faults which may occur during the operation of the MEMS. These faults can be detected by implementing different techniques such as residuals, which are signals representing the difference between the nominal behavior of the MEMS and its actual behavior. If this difference is greater than a predefined value, then the MEMS is subject to abnormal situation.

- Fault threshold: this is an important parameter needed to estimate the RUL. Fault thresholds can be obtained by different ways. They can be given by experts working on the studied MEMS, obtained statistically by exploiting the knowledge gathered from the experience feedback, defined theoretically through the utilization of formulas related to failure mechanisms or experimentally by observing the response of the MEMS when performing accelerated life tests. However, whatever the technique used, fault thresholds are related to the MEMS performance variation. This performance, and consequently the fault threshold, can be related to the stability of the MEMS, its precision, its time response, etc. The thresholds can then be obtained by setting acceptable performance values. 
- RUL estimation: the time to failure is calculated as the difference between the failing time and the current time, as illustrated in figure 4 .

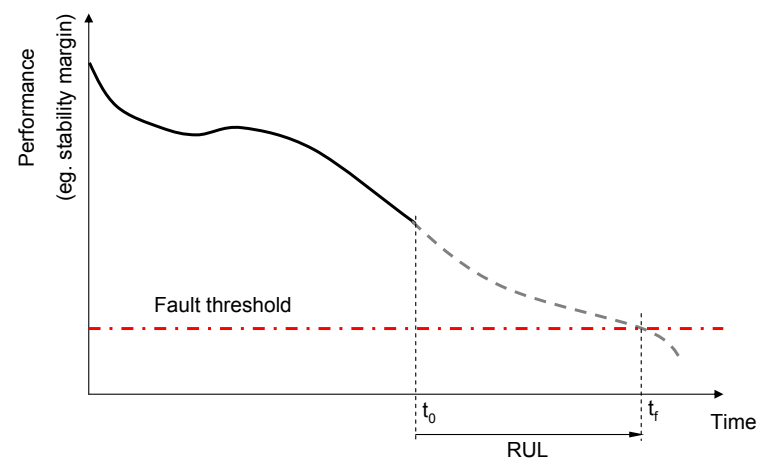

Figure 4: Illustration of fault threshold.

\section{Case study and simulation results}

The method presented in section 3 is applied on a MEMS consisting of a microgripper. The steps of the method are implemented and explained in the following of the paper.

\subsection{Description of the experiments}

The experimental platform used to test the proposed method is shown in figure 5 and its global synoptic is illustrated by figure 6 .

The central component of the platform consists of a FT-G100 force sensing microgripper of "Femto tools" company designed to handle micro and nanoobjects. The initial opening of the microgripper arms is $100 \mu \mathrm{m}$ and can be controlled with nano-meter precision. The maximum actuation voltage of the microgripper is $200 \mathrm{~V}$. A square signal of $5 \mathrm{~V}$ magnitude and frequency equal to $25 \mathrm{~Hz}$ is generated by an ARDUINO device, the signal is then amplified and supplied to the microgripper. The actuation is monitored by measuring the displacement of its mobile arm through an interferometer (due to the design of the microgripper, only one of its two arms is moving, the second is static). The interferometer points a laser beam through a head to the mobile arm and calculates the displacement by processing the reflected signal. The quality of the measurements depend on the amount of the laser beam reflected by the mobile arm. This quality is optimized by placing the 


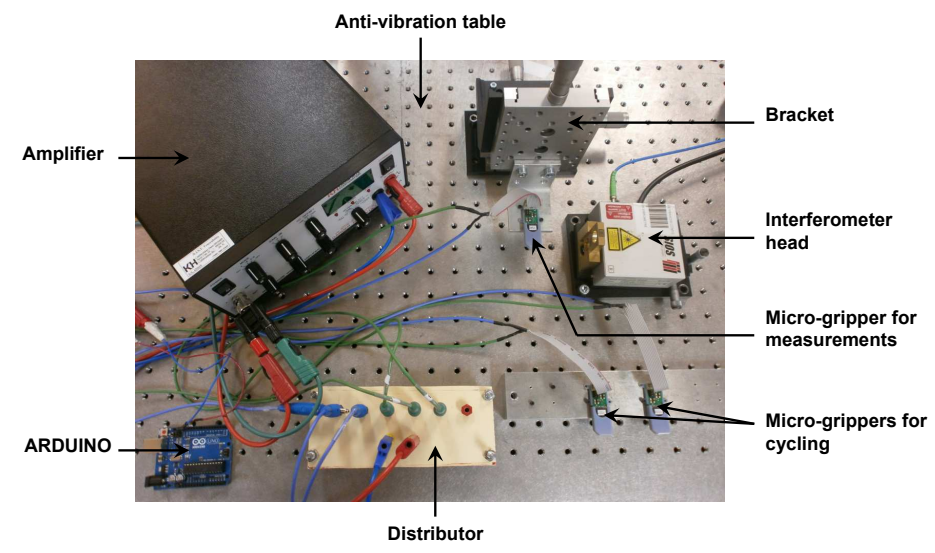

Figure 5: Overview of the experimental platform.

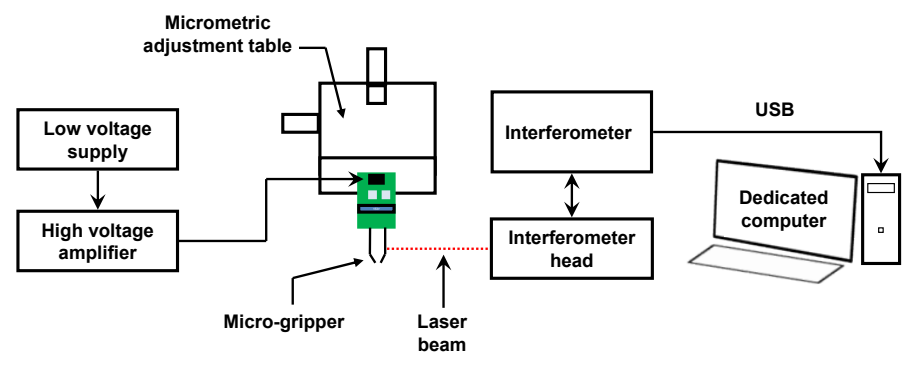

Figure 6: Global synoptic of the platform.

head generating the laser beam at a distance equal to $5 \mathrm{~cm}$ from the mobile arm and by adjusting the alignment of the beam with the mobile arm by a mean of a three directions $(x, y, z)$ table. The reflected signal is then acquired at a frequency equal to $25 \mathrm{kHz}$, with 16384 points at each acquisition, and stored in different files in a dedicated computer for later use.

\subsection{Nominal behavior model}

The microgripper shown in figure 7 can be assimilated to a second order dynamic system whose physical scheme is shown in figure 8 . The application of the second fundamental law of dynamics leads to the following equation:

$$
F-f . \dot{x}-k . x=M . \ddot{x}
$$

where $F$ is the electrical force actuating the mobile arm and which is proportional to the input voltage $[28,29] V(t): F=\frac{N_{a} \cdot \varepsilon \cdot h_{z}}{2 . g} \cdot V^{2}(t), N_{a}=1300$ 

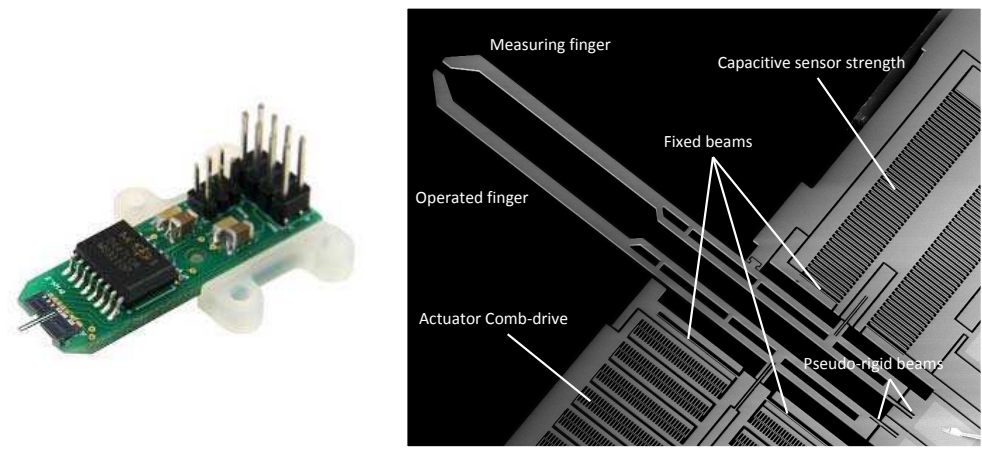

Figure 7: The microgripper FT-G100 used in the accelerated tests.

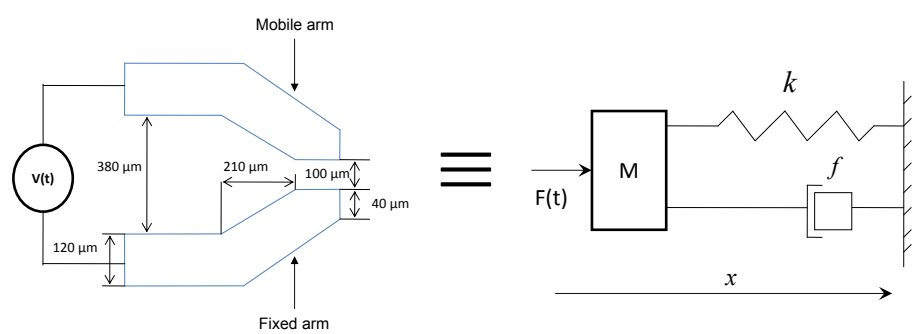

Figure 8: Physical scheme of the microgripper.

is the number of electrodes of the comb drive, $\epsilon=8.85 \mathrm{pF} / \mathrm{m}$ is the air permittivity, $h_{z}=50 \mu \mathrm{m}$ is the thickness of the electrodes and $\mathrm{g}=6 \mu \mathrm{m}$ is the gap between the fixed and the mobile electrodes of the comb drive, $f$ is the friction coefficient, $k$ is the stiffness of the arm and $M$ is its mass. By applying the Laplace transform on equation (1), and by putting $\eta=\frac{N_{a} \cdot \varepsilon \cdot h_{z}}{2 \cdot g}$ and $U(t)=V^{2}(t)$, one gets the canonical transfer function given in equation (2) (which is in the form of a second order model) of the microgripper. The canonical equation of a second order dynamic system, its time response and its characteristic values (settling time, rising time, overshoot, etc.) are well detailed and explained in the following references: [30] (chapter 3, subsections 3.3 and 3.4) and [31] (chapter 6).

$$
H(p)=\frac{X(p)}{U(p)}=\frac{\frac{\eta}{k}}{1+\frac{f}{k} p+\frac{M}{k} p^{2}}=\frac{K}{1+\frac{2 \xi}{\omega_{n}} p+\frac{1}{\omega_{n}^{2}} p^{2}}
$$

In equation (2) $K=\frac{\eta}{k}$ is the static gain of the microgripper, $\omega_{n}=\sqrt{\frac{k}{M}}$ its natural frequency and $\xi=\frac{1}{2} \cdot \frac{f}{\sqrt{k \cdot M}}$ its dumping coefficient. 
The time response obtained experimentally from a new microgripper is

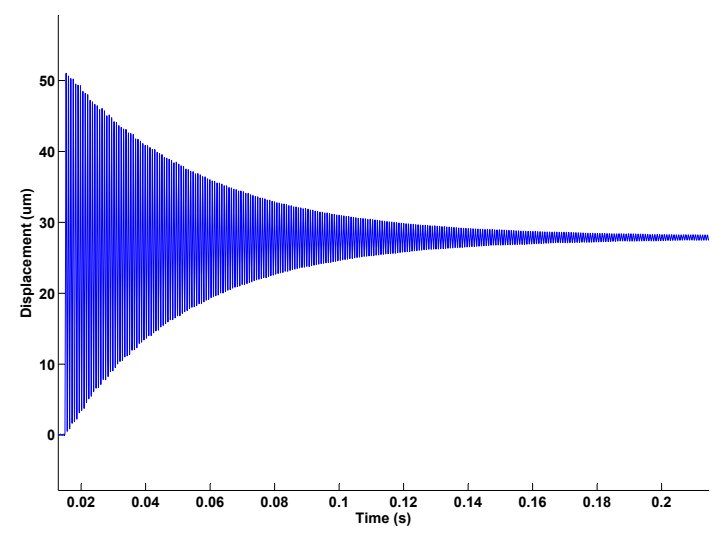

Figure 9: Time response of the microgripper.

shown in figure 9. This time response corresponds to a second order dynamic system for which the numerical values of $K, \xi$ and $\omega_{n}$ can be obtained by using the overshoot $D \%=100 \times e^{-\left(\frac{\pi \xi}{\sqrt{1-\xi^{2}}}\right)}$ and the pseudo period $T_{p}=\frac{2 \pi}{\omega_{n} \sqrt{1-\xi^{2}}}$ formulas. These latter formulas can be extracted from the time response equation of the microgripper by solving the differential equation given in (1) (more details on the time response of a second order system, its overshoot and pseudo period can be found in [30], chapter 3, subsections 3.3 and 3.4 and in [31], chapter 6). The values of $K, \xi$ and $\omega_{n}$ estimated from the time response of figure 9 are equal to $0.341 \mu \mathrm{m} / V^{2}, 0.06$ and $362.5 \mathrm{rad} / \mathrm{s}$, respectively.

\subsection{Degradation models}

The degradation of the microgripper can be related to drifts of its physical parameters, which then affect its static and dynamic performances. According to equation (1), the parameters which can vary are the mass $M$, the friction coefficient $f$ and the stiffness $k$. In practice, the variation of the two first parameters can be neglected. Thus, only the stiffness can vary significantly due to cyclings. To verify this assumption, we have designed the experimental platform shown in figure 5 where three microgrippers are continuously cycled and their behaviors are acquired at a given sampling frequency as shown in figure 10 .

In this application the cycling frequency is equal to $\frac{1}{T}=\frac{1}{0.04}=25 \mathrm{~Hz}$ 


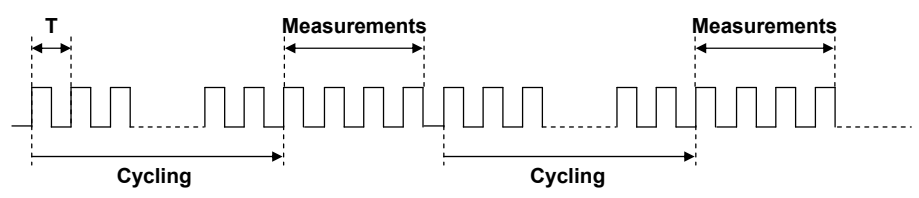

Figure 10: Cycling and measurements during the accelerated life tests.

and the measurements are done each 2160000 cycles. These values are defined according to the total number of cycles the microgrippers can perform and which are guaranteed by the manufacturer: no performance degradation over 100 million cycles. By considering this last information, one has to wait 46 days before observing significant drift of the performance. To show the feasibility of the proposed method, and while waiting to have complete experimental data, we have considered different simulated degradations of the microgrippers represented by linear and nonlinear models of the stiffness $k$. Furthermore, to be close to the behavior of the cycled devices, the initial value of $k$ in the simulated degradation models is set equal to the numerical value identified from the time response of the microgripper: $k=11 \mathrm{~N} / \mathrm{m}$. Examples of linear, polynomial and exponential degradation models used in the following simulations are given by equations (3), (4) and (5), respectively. In each simulated degradation model, the values of the coefficients can be varied to simulate different trends and get closer to the reality.

$$
\begin{gathered}
k(t)=-0.95 t+11 \\
k(t)=-t^{2}+t+11 \\
k(t)=11 \mathrm{e}^{-t}
\end{gathered}
$$

\subsection{Condition assessment and RUL estimation}

The substitution of the degradation models, represented by the variation of the parameter $k$ and given in equations (3), (4) and (5), in the behavior model of the microgripper given in equation (2) leads to a global model where the static and dynamic performances of the microgripper are time dependent. The health state of the microgripper can then be assessed at each time by analyzing its time response for different degradation models. Furthermore, the RUL of each microgripper can be calculated at each time. To do this, one needs to define a performance threshold (which can be also seen as a fault threshold) according to different criteria: stability of the microgrippers, their 
precision, their settling time, etc. By analyzing the time response of these devices for different values of $k$, one can observe that they remain always stable. However, their settling time $t_{s}$ increases as the stiffness decreases. Indeed, this relation can be verified through equation (6) (more details can be found in [30], chapter 3, subsections 3.3 and 3.4 and in [31], chapter 6):

$$
t_{s}=-\frac{1}{\xi \omega_{n}} \ln \left(0.05 \sqrt{1-\xi^{2}}\right)
$$

To set the numerical value of the performance threshold, one can rely on the settling time of a new microgripper, which can be calculated from the time response given in figure 9 and which is equal in this case to $0.1378 \mathrm{~s}$. We can then suppose that if the settling time exceeds $n \times t_{s}$, with $n \gg 1$, the microgripper can be considered as out of service and its RUL can be calculated as the remaining time before reaching this performance threshold. In this application, the value of $n$ is defined arbitrary, but in practice it should be set by the user according to the level of performance he/she wants to reach.

Figures 11, 12 and 13 show some simulation examples related to different
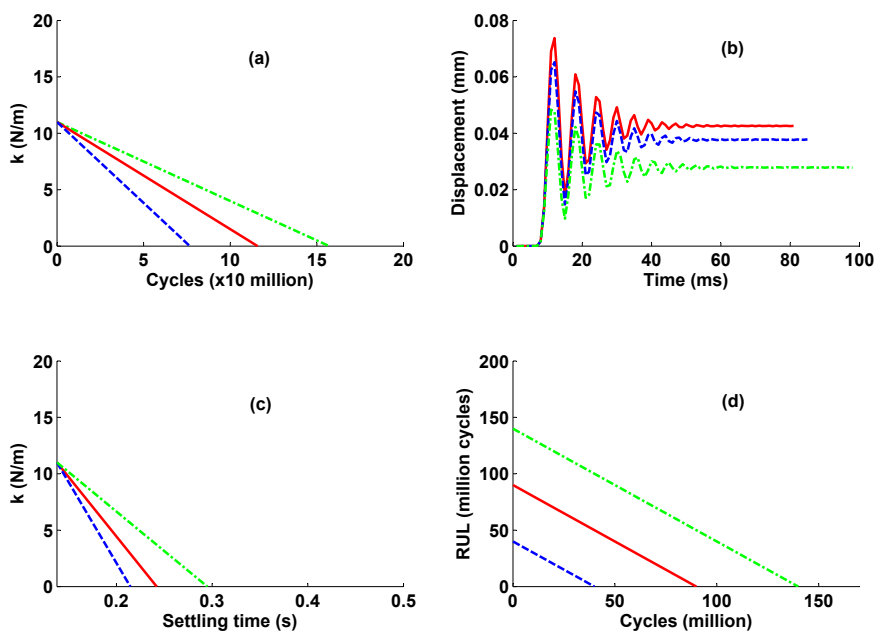

Figure 11: Simulation results related to linear degradation models.

stiffness degradations (linear, exponential and polynomial) and their impact on the time response and on the estimated RUL. The sub-figures of each one of these figures concern: a) the variation of stiffness $k$ versus number of cycles, b) the time response, c) the variation of stiffness versus settling time and d) RUL estimation according to the defined thresholds. For example, 

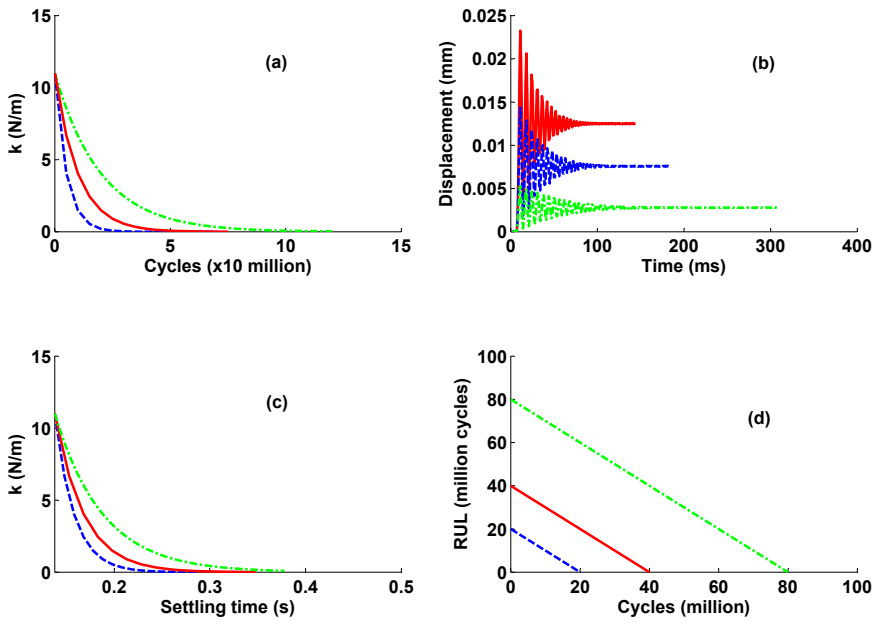

Figure 12: Simulation results related to exponential degradation models.
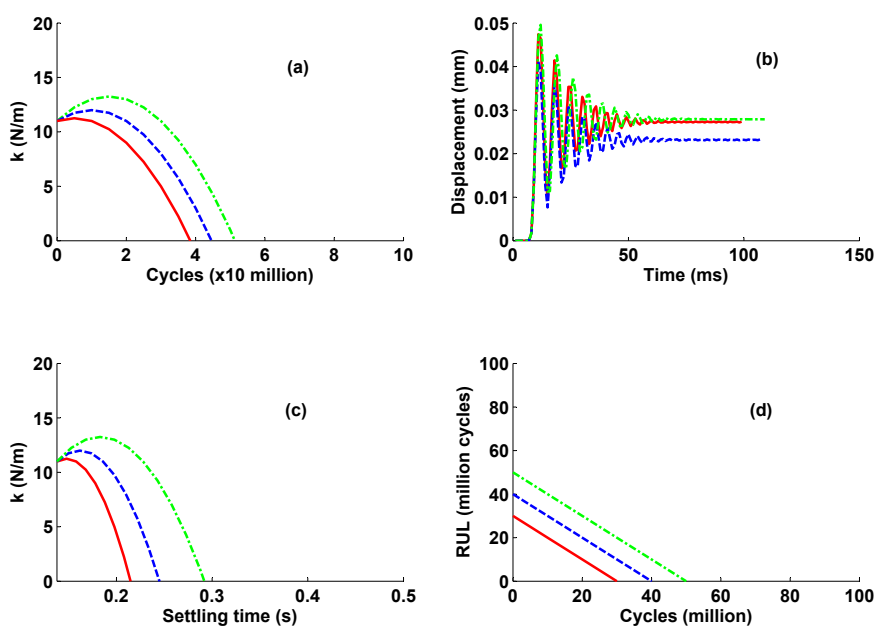

Figure 13: Simulation results related to polynomial degradation models.

the settling time thresholds defined for the exponential degradation are: 0.2 $s$ for the blue line, $0.25 s$ for the red line and $0.3 s$ for the green line.

From these figures, one can observe that the variation of the stiffness $k$ impacts the dynamics of the microgripper (sub-figures (b)) and its estimated RUL (sub-figures (d)). Indeed, the estimated RULs are coherent with the types and trends of the degradations. For example, in all simulated cases, the RUL depends on the severity of the degradation: "quick" degradations 
will lead to short RULs. In addition to RUL estimation, the state of the microgripper can be assessed and predicted at each time. This information can then be used to take appropriate decisions, such as control laws reconfiguration, to maintain as long as possible the function for which the microgripper is dedicated. We can for example imagine a set of microgrippers, each one manipulating a specific component, in the purpose of assembling desired micro-systems. It is then possible to monitor, assess and estimate the RUL of each microgripper in order to guarantee free faults and high quality of the final assembled micro-systems.

\section{Conclusion}

A condition assessment and fault prognostic method with application to MEMS is presented in this paper. First, a brief state of the art of MEMS reliability and related failure mechanisms is given. The analysis of the reported works allowed identification of some challenges to tackle before implementing PHM algorithms and methods.

The proposed method is then presented and its steps explained. The method relies on two models: a nominal behavior model of the MEMS and degradation models related to its components, which are subject to failures. Both models are then merged and used to assess the health state of the targeted MEMS and predict its RUL. The calculation of this latter information requires the definition of a performance threshold (which can be also seen as a failure threshold) beyond which the MEMS is considered faulty or out of service. To do this, we have proposed some criteria to use (precision, stability, time response, etc.).

The method can be applied on different categories of MEMS at a condition that the assumptions set before presenting the method hold. In this contribution the case of microgrippers is addressed. The simulation results showed the effectiveness of the method on such devices. However, its application on real degradations learned from the studied MEMS would increase its relevance. Finally, it has to be noted that the results of condition assessment and RUL estimation can be used to take appropriate decisions to accommodate the faults or delay the failure time.

\section{References}

[1] A. K. Jardine, D. Lin, D. Banjevic, A review on machinery diagnostics and prognostics implementing condition-based maintenance, Mechanical 
Systems and Signal Processing 20 (7) (2006) 1483 - 1510.

[2] A. Heng, S. Zhang, A. C. Tan, J. Mathew, Rotating machinery prognostics: State of the art, challenges and opportunities, Mechanical Systems and Signal Processing 23 (3) (2009) $724-739$.

[3] K. Medjaher, D. A. Tobon-Mejia, N. Zerhouni, Remaining useful life estimation of critical components with application to bearings, IEEE Transactions on Reliability 61 (2) (2012) $292-302$.

[4] J. Sikorska, M. Hodkiewicz, L. Ma, Prognostic modelling options for remaining useful life estimation by industry, Mechanical Systems and Signal Processing 25 (2011) 1803 - 1836.

[5] D. Tanner, MEMS reliability: Where are we now?, Microelectronics Reliability 49 (2009) $937-940$.

[6] Y. Huang, A. S. S. Vasan, R. Doraiswami, M. Osterman, M. Pecht, MEMS reliability review, IEEE TRANSACTIONS ON DEVICE AND MATERIALS RELIABILITY 12 (2) (2012) 482 - 493.

[7] U. Zaghloul, G. Papaioannou, B. Bhushan, F. Coccetti, P. P. R. Plana, On the reliability of electrostatic NEMS/MEMS devices: Review of present knowledge on the dielectric charging and stiction failure mechanisms and novel characterization methodologies, Microelectronics Reliability 51 (2011) 1810 - 1818.

[8] W. M. Van Spengen, MEMS reliability from a failure mechanisms perspective, Microelectronics Reliability 43 (2003) 1049 - 1060.

[9] Y. Li, Z. Jiang, Handbook of Performability Engineering, Springer, 2008, Ch. An Overview of Reliability and Failure Mode Analysis of Microelectromechanical Systems (MEMS), pp. 953 - 966.

[10] M. Matmat, F. Coccetti, A. Marty, R. Plana, C. Escriba, J.-Y. Fourniols, D. Esteve, Capacitive RF MEMS analytical predictive reliability and lifetime characterization, Microelectronics Reliability 49 (2009) $1304-$ 1308 .

[11] B. Wunderle, B. Michel, Progress in reliability research in the micro and nano region, Microelectronics Reliability 46 (2006) 1685 - 1694. 
[12] V. T. Srikar, S. D. Senturia, The reliability of microelectromechanical systems (MEMS) in shock environments, JOURNAL OF MICROELECTROMECHANICAL SYSTEMS 11 (3) (2002) 206 - 214.

[13] D. J. Fonseca, M. Sequera, On MEMS reliability and failuremechanisms, International Journal of Quality, Statistics, and Reliability (2011) 7 pagesdoi:10.1155/2011/820243.

[14] B.-L. Chen, P.-C. Huang, L.-S. Jang, M.-K. Chen, Electrical failure analysis of peristaltic micropumps fabricated with PZT actuators, Microelectronics Reliability 52 (2012) 1080 - 1085.

[15] G. Langfelder, A. Longoni, F. Zaraga, A. Corigliano, A. Ghisi, A. Merassi, A new on-chip test structure for real time fatigue analysis in polysilicon MEMS, Microelectronics Reliability 49 (2009) 120 126.

[16] G. Zhang, C. Volkert, R. Schwaiger, R. Mönig, O. Kraft, Fatigue and thermal fatigue damage analysis of thin metal films, Microelectronics Reliability 47 (2007) 2007 - 2013.

[17] R. Asgary, K. Mohammadi, M. Zwolinski, Using neural networks as a fault detection mechanism in MEMS devices, Microelectronics Reliability 47 (2007) $142-149$.

[18] J. Walraven, Failure analysis issues in microelectromechanical systems (MEMS), Microelectronics Reliability 45 (2005) 1750 - 1757.

[19] S. Mellé, D. D. Conto, L. Mazenq, D. Dubuc, B. Poussard, C. Bordas, K. Grenier, L. Bary, O. Vendier, J. Muraro, J. Cazaux, R. Plana, Failure predictive model of capacitive RF-MEMS, Microelectronics Reliability 45 (2005) $1770-1775$.

[20] G. Langfelder, A. Longoni, F. Zaraga, Monitoring fatigue damage growth in polysilicon microstructures under different loading conditions, Sensors and Actuators A 159 (2010) 233 - 240.

[21] S. MIR, B. CHARLOT, B. COURTOIS, Extending fault-based testing to microelectromechanical systems, JOURNAL OF ELECTRONIC TESTING: Theory and Applications 16 (2000) 279 - 288. 
[22] M. McMahon, J. Jones, A methodology for accelerated testing by mechanical actuation of MEMS devices, Microelectronics Reliability 52 (2012) 52 (2012) $1382-1388$.

[23] M. Matmat, K. Koukos, F. Coccetti, T. Idda, A. Marty, C. Escriba, J.-Y. Fourniols, D. Esteve, Life expectancy and characterization of capacitive RF MEMS switches, Microelectronics Reliability 50 (2010) 1692 - 1696.

[24] J. Ruan, N. Nolhier, G. Papaioannou, D. Trémouilles, V. Puyal, C. Villeneuve, T. Idda, F. Coccetti, R. Plana, Accelerated lifetime test of RF-MEMS switches under esd stress, Microelectronics Reliability 49 (2009) $1256-1259$.

[25] D. Tanner, T. Parson, A. Corwin, J. Walraven, J. Wittwer, B. Boyce, S. Winzer, Science-based MEMS reliability methodology, Microelectronics Reliability 47 (2007) $1806-1811$.

[26] W. Van Driel, D. Yang, C. Yuan, M. van Kleef, G. Zhang, Mechanical reliability challenges for MEMS packages: Capping, Microelectronics Reliability 47 (2007) 1823 - 1826.

[27] H. Tilmans, J. D. Coster, P. Helin, V. Cherman, A. Jourdain, P. D. Moor, B. Vandevelde, N. Pham, J. Zekry, A. Witvrouw, I. D. Wolf, MEMS packaging and reliability: An undividable couple, Microelectronics Reliability 52 (2012) 2228 - 2234 .

[28] V. A. L. de Almeida, P. H. de Godoy, E. C. N. Silva, R. C. Ibrahim, Microgrippers driven by electrostatic comb drive actuators, ABCM Symposium Series in Mechatronics 1 (2004) 682 - 687.

[29] M. Boudaoud, Caractéristique dynamique des bruits à l'échelle nanométrique et commande robuste LVP de systèmes de micromanipulation, Ph.D. thesis, U.F.R des sciences et techniques de l'université de Franche-Comté (2012).

[30] G. F. Franklin, J. D. Powell, A. Emami-Naeini, Feedback control of dynamic systems, Pearson Prentice Hall, 2006.

[31] D. Rowell, D. N. Wormley, System dynamics: An Introduction, Prentice Hall, 1997. 